\title{
Mouse Prostate-Specific Membrane Antigen Plasmid DNA Vaccine
}

National Cancer Institute

\section{Source}

National Cancer Institute. Mouse Prostate-Specific Membrane Antigen Plasmid DNA

Vaccine. NCI Thesaurus. Code C48411.

A vaccine consisting of a plasmid DNA encoding the murine prostate-specific membrane antigen (PSMA). Upon administration, expressed PSMA may stimulate a cytotoxic T cell response against tumor cells that express PSMA, resulting in tumor cell lysis. ( $\mathrm{NCI05)}$ 